\title{
Development of Miniaturized Fiber-Optic Laser Doppler Velocimetry Sensor for Measuring Local Blood Velocity: Measurement of Whole Blood Velocity in Model Blood Vessel Using a Fiber-Optic Sensor with a Convex Lens-Like Tip
}

\author{
Tsutomu Tajikawa, ${ }^{1}$ Wataru Ishihara, ${ }^{2}$ Shimpei Kohri, ${ }^{3}$ and Kenkichi Ohba ${ }^{1}$ \\ ${ }^{1}$ Department of Mechanical Engineering, Kansai University, Osaka 564-8680, Japan \\ ${ }^{2}$ Boston Scientific, Tokyo 160-0023, Japan \\ ${ }^{3}$ Department of Medical Engineering, Aino University Osaka 564-8680, Japan
}

Correspondence should be addressed to Tsutomu Tajikawa, tajikawa@kansai-u.ac.jp

Received 2 December 2011; Accepted 13 April 2012

Academic Editor: Jinsong Leng

Copyright (C) 2012 Tsutomu Tajikawa et al. This is an open access article distributed under the Creative Commons Attribution License, which permits unrestricted use, distribution, and reproduction in any medium, provided the original work is properly cited.

\begin{abstract}
A miniaturized fiber-optic laser Doppler velocimetry sensor has been developed to measure the local blood velocity in vivo. The laser beam emitted from the sensor tip can be focused at any distance between 0.1 and $0.5 \mathrm{~mm}$ from the tip. Consequently, the sensor has a sufficiently high signal-to-noise ratio to measure the local velocity in almost any opaque fluid, including blood. The sensor head is inserted in an injection needle or a catheter tube. In the former case, it is inserted at an angle to the wall of a vessel and is scanned across the vessel to measure the velocity distribution. In the latter case, it is aligned parallel with the flow in a vessel. For all flows of whole human blood, whole caprine blood, and $69 \%$ hematocrit of bovine blood, the velocity distribution across the vessel could be measured very accurately. The insertion angle of the fiber into the flow significantly affects the measurement accuracy; an angle of about $50^{\circ}$ is suitable when an injection needle is used. When a catheter is employed, an insertion direction opposite to the flow direction is better than parallel to the flow due to the generation of a wake behind the fiber.
\end{abstract}

\section{Introduction}

Tanaka and Benedek [1] first developed a fiber-optic laser Doppler velocimetry (LDV) sensor. Kajiya et al. improved it and used it to measure the local blood flow velocity in animal testing [2]. However, the fiber tip transmitted and received light inefficiently, and a dead flow region was observed in front of the fiber tip. Furthermore, it was difficult to observe the dominant frequency in the spectrum of the signal as a function of the Doppler frequency shift. Consequently, this technique has yet to be applied in diagnosis devices.

One of the authors has developed several fiber-optic LDV probes including a probe with an optical dual-fiber pickup that separates the transmission and receiving optical components by using two optical fibers placed alongside each other [3] and a probe with a truncated cone-shaped distributed index lens (diameter: $1 \mathrm{~mm}$ ) [4, 5]. However, the flow field is disturbed when the sensor head is directly inserted into the working fluid and the signal-to-noise ratio of the Doppler signal is low due to strong reflection at the interface between the fiber tip and the distributed index lens. Consequently, it was very difficult to measure the flow velocity accurately. To reduce the flow disturbance caused by the probe and to improve the quality of the Doppler signal obtained by heterodyne interferometry, we have been developing a minimized fiber-optic LDV probe with a convex lens at the fiber tip fabricated by microelectromechanical systems technology [6].

This paper reports the development of a fiber-optic LDV system by using an etched fiber with a convex lens-like tip and evaluates the performance of the system by measuring local blood flow velocity. 


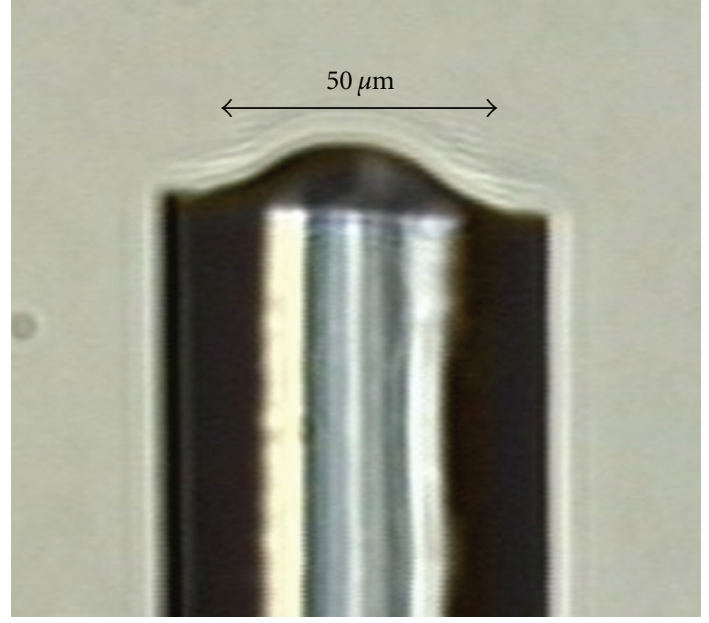

Figure 1: Chemically etched quartz fiber probe. ( $\mathrm{HF}: \mathrm{NH}_{4} \mathrm{~F}: \mathrm{H}_{2} \mathrm{O}$ $=1: 7: 5, T=5.0 \mathrm{~h}$ ).

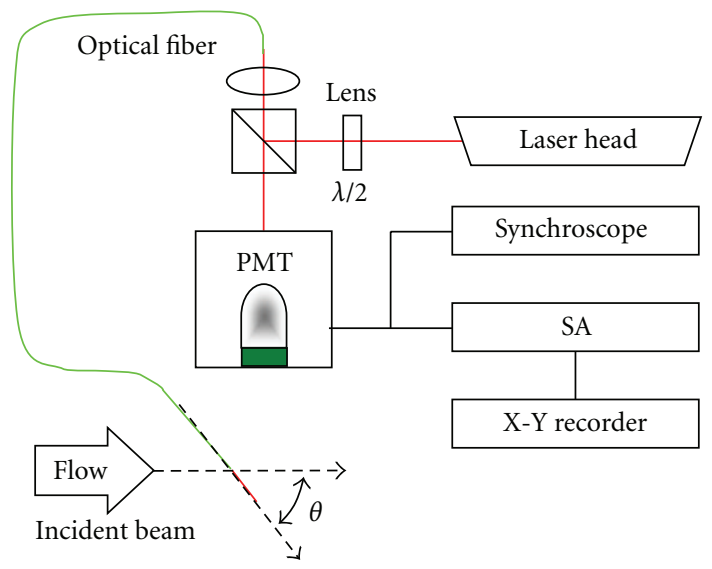

Figure 2: Schematic diagram of fiber-optic LDV system.

\section{Experimental Setup and Methods}

2.1. Fiber-Optic LDV System [6]. Convex lens-like fiber tips were fabricated by chemical etching. Specifically, multimode quartz fibers with a graded refractive index were etched in aqueous solutions of hydrogen fluoride and ammonium fluoride. The solution concentration, etching time, and etching temperature were controlled to obtain the desired radius of curvature for the lens-like surface of the fiber tip (see Figure 1). An optical fiber etched under optimal conditions was used to produce a fiber-optic LDV system for measuring the local blood velocity (see Figure 2).

This system employs a reference beam. A monochromatic beam from a helium-neon laser $(\lambda=632.8 \mathrm{~nm}$; power: $25 \mathrm{~mW}$ ) that is perpendicularly polarized (S polarized) after passing through a half wave plate is reflected by a polarized beam splitter (PBS). The reflected beam is focused by a lens on the tip of the optical fiber (Sumitomo Electric Industries, Ltd., EG-5; core diameter: $50 \mu \mathrm{m}$; cladding diameter: $125 \mu \mathrm{m}, N A=0.21$ ). The laser beam passes through the optical fiber and is emitted from the convex

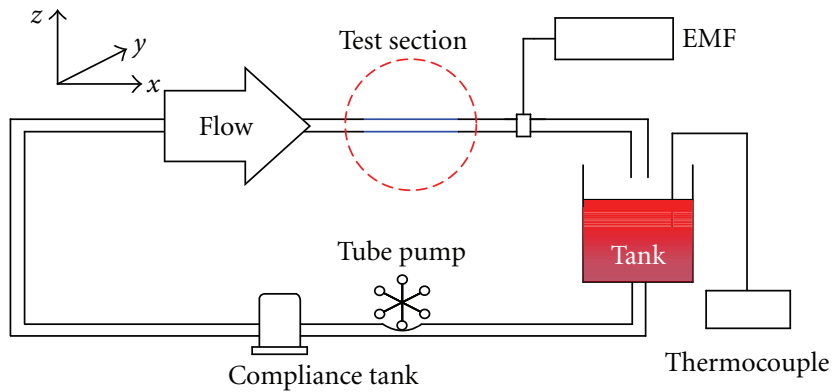

Figure 3: Schematic diagram of model flow system.

lens-like fiber end. It converges at the focal point where the measurement volume is formed, because the intensity is maximized at that point. Ray optics calculations based on a microscopy image of the fiber tip surface predict that the laser beam in the optical fiber will converge at a distance of about $L=220 \mu \mathrm{m}$ from the fiber tip. Therefore, the measurement volume (beam waist, $d_{0}: 3.3 \mu \mathrm{m}$; focal depth, $\left.l_{0}: 37 \mu \mathrm{m}\right)$ is formed. The light intensity at the focal point is about 160 times higher than that of the incident light on the fiber. When scattering particles, such as, erythrocytes pass through the focal point, they scatter the incident light and shift its frequency by the Doppler effect. Back-scattered light is collected by the same fiber, whereas parallel polarized (P-polarized) light consisting of scattered light and light partially reflected at the fiber end, which functions as a local oscillator, pass through the PBS. Superimposing the scattered and local oscillator light generates heterodyne interference. The light is detected by a photomultiplier tube (PMT; Hamamatsu Photonics K.K., R1477-06). The Doppler signal detected by heterodyne interferometry is processed by a commercial spectrum analyzer (SA) and recorded by an X$\mathrm{Y}$ recorder. The peak in each spectrum is taken to be the Doppler frequency. When a clear peak is not observed in a spectrum, the velocity is either not measured or set to zero. The sensor probe is inserted obliquely into the fluid at an angle $\theta$ between the fiber axis and the flow direction. The relationship between the Doppler frequency and the flow velocity is given by

$$
u=\frac{f_{D} \cdot \lambda}{2 n \cos \theta}
$$

$n$ is the refractive index of working fluid.

2.2. In-Vitro Circulation Flow Circuit. Figure 3 shows a schematic diagram of the experimental setup for an invitro circulation flow circuit. This circuit consists of a closed loop and has a reservoir, a tube pump, a compliance tank for removing flow pulsations, and a silicone rubber tube (inner diameter: $6 \mathrm{~mm}$; outer diameter: $8 \mathrm{~mm}$, length: $1 \mathrm{~m}$ ) to produce a well-developed flow field. This system was used as a model cardiovascular system. The volumetric flow rate and working fluid temperature were measured by an electromagnetic flowmeter (EMF) and a thermocouple, respectively. 


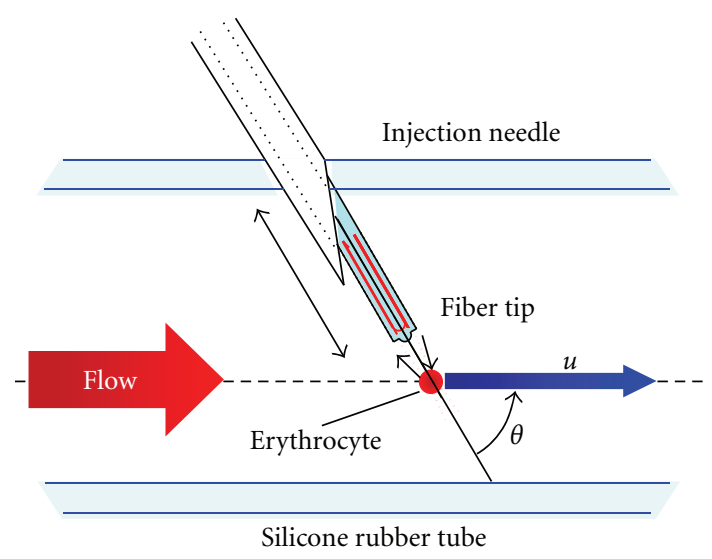

(a) Needle injection system

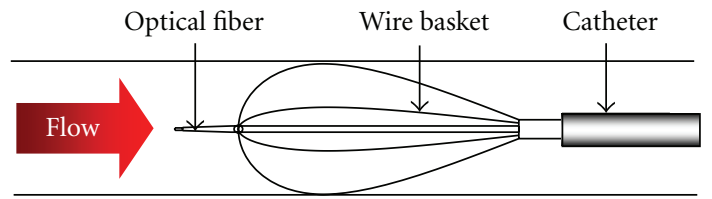

(b) Catheter injection system

FIgURE 4: Schematic diagram of LDV probe injection system.

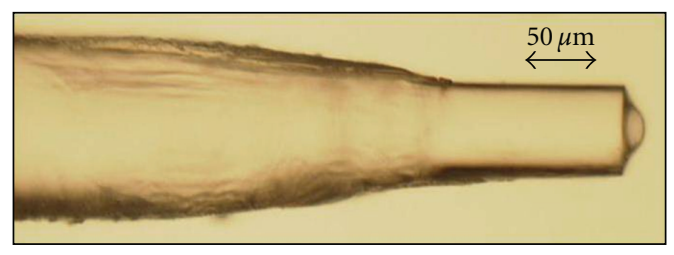

(a) Tapered optical fiber LDV sensor

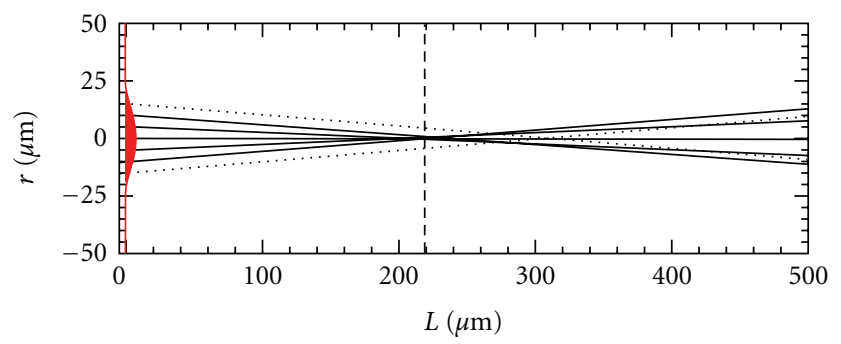

(b) Light path of collimated beam from etched fiber tip in PBS

FIGURE 5: Chemically etched quartz fiber tip as LDV sensor.

2.3. Fiber-Optic LDV Probe Insertion Method. Two insertion methods were employed to introduce the fiber-optic LDV probe to the measurement point (see Figure 4).

The first method used an insertion needle with an outer diameter of $0.5 \mathrm{~mm}$. The fiber-optic sensor probe was obliquity inserted into the test section, and it was moved along the needle using a micromotion slide stage to measure the velocity profile in the tube. Prior to the measurement, the Doppler frequency was measured at the center of the tube to evaluate the measurement capability, and the sensor probe was moved along the tube diameter to find a place on the tube wall where there was no clear peak in the spectral waveform. After detecting the sensor position, the velocity distribution in the tube was measured.

A second method to enable the sensor probe to be used in clinical applications, such as, interventional devices. In this method, the fiber-optic sensor was incorporated into a catheter. A catheter for cardiovascular interventional treatment was used to introduce the fiber tip to the measurement point. This catheter (Wilson-Cook Medical Inc., 3.5-Fr; outer diameter: $1.17 \mathrm{~mm}$ ), which is designed to remove foreign bodies in clinical applications, was inserted either upstream $\left(\theta=180^{\circ}\right)$ or downstream $\left(\theta=0^{\circ}\right)$ of the test section. The fiber tip with a folding $\mathrm{Ni}$-Ti wire frame was pushed down the catheter tube to the measurement point, and the frame was extended to fix the probe at the center of the tube.

\section{Experimental Results and Discussion}

3.1. Measurement of Velocity Profile in Human Blood Flow. The flow velocity profiles of whole human blood and a white pigment suspension, which was used as a semiopaque and opaque fluid in previous studies [4-6], were measured in the model blood vessel using the etched fiber probe. An optical fiber with a convex lens-like tip (etched using a volumetric concentration ratio of the buffer solutions with $\mathrm{HF}: \mathrm{NH}_{4} \mathrm{~F}: \mathrm{H}_{2} \mathrm{O}=1: 7: 5$ for $4 \mathrm{~h}[6]$ ) was used (see Figure 5). A red cell concentrate in a mannitol-adeninephosphate solution (provided by the Japanese Red Cross Osaka Blood Center) was diluted with phosphate-buffered saline (PBS) to give $H_{\mathrm{ct}}=45 \%$, which is the same as that of human whole blood. The insertion angle was set to $\theta=80^{\circ}$ and flow rates through the model vessel of $0.2,0.4$, and $0.6 \mathrm{~L} / \mathrm{min}$ were used. Because blood plasma mostly consists of water (about $93 \%$ by volume), plasma is considered to have a similar refractive index to PBS. Therefore, the refractive index of saline was used to estimate the measurement volume. Ray optics calculations using a microscopy image of the fiber tip surface predict that the laser beam from the lens-like fiber tip will converge at a distance of $220 \mu \mathrm{m}$ from the tip in PBS with a refractive index of 1.333 and for a focal length at a $220 \mu \mathrm{m}$ [6].

To prevent reductions to the fiber diameter and fiber strength during chemical etching when fabricating the 

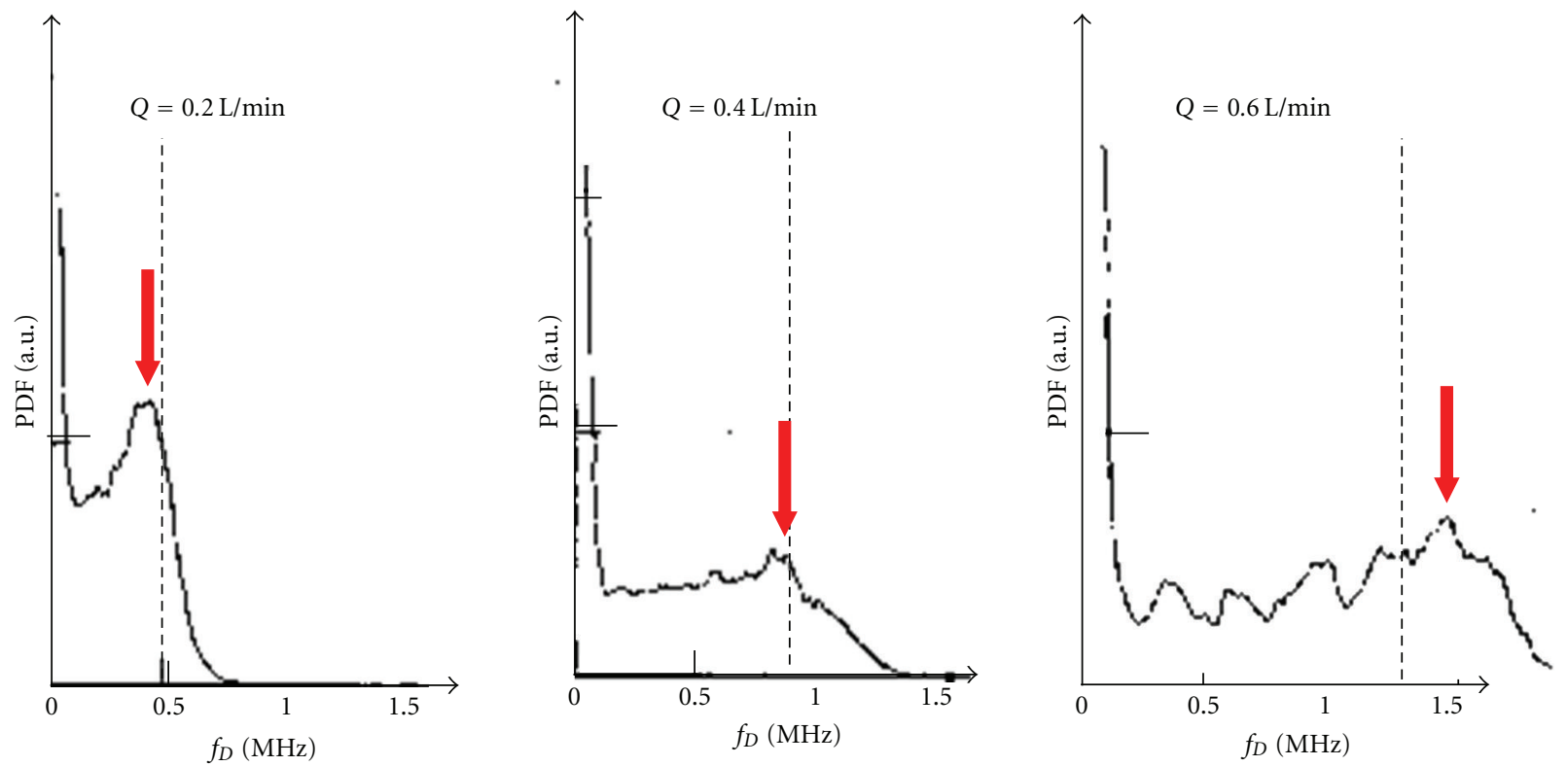

FIGURE 6: PDFs of Doppler signal for various flow rates.

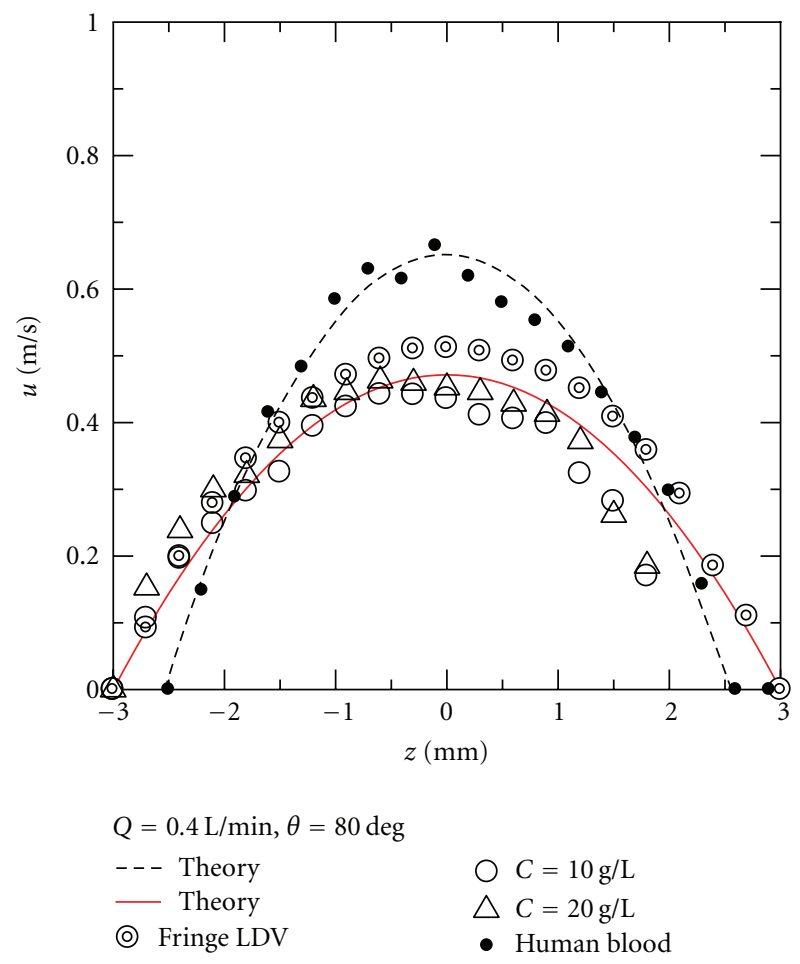

FIgURE 7: Velocity profiles in model blood vessel.

convex lens-like fiber tip, the fiber sidewall was coated with a photoresist (Tokyo Ohka Kogyo Co. Ltd., OFPR800). Figure 6 shows examples of the frequency spectrum of the time-averaged Doppler signal, and Figure 7 shows velocity profiles measured in model vessels at a volumetric flow rate of $Q=0.4 \mathrm{~L} / \mathrm{min}$. The vertical axis in Figure 6 shows the probability density function (PDF) of the time-averaged
Doppler signal while the horizontal axis shows the frequency. The vertical broken lines in Figure 6 indicate the Doppler frequency corresponding to the axial flow velocity, which is calculated from the relationship between the volumetric flow rate and the parabolic velocity profile of laminar Poiseuille flow. The parabolic curves in Figure 7 show the velocity profiles for Poiseuille flow calculated from the volumetric flow rate. The solid and dashed lines are the theoretical velocity profiles in tubes with diameters of 6.0 and $5.1 \mathrm{~mm}$, respectively, and the double circle points indicate the water flow velocity profiles measured using the dual beam mode (fringe-type) LDV system.

These results clearly show that the signal-to-noise ratio of the Doppler signal decreases with increasing volumetric flow rate. This reduction is considered to be caused by a change in the flow pattern as the flow changes from being laminar to being turbulent. Despite this reduction in the signal-tonoise ratio, the dominant frequency corresponding to the Doppler shift was observed as a peak in all the frequency spectra obtained in this experiment.

Because the injection needle used to insert the fiber optic LDV probe locally disturbed the flow field, the local flow velocity at $z=3 \mathrm{~mm}$ (i.e., on the surface of the upper wall) could not be measured accurately. By assuming Poiseuille flow and a tube diameter of $5.1 \mathrm{~mm}$ which is suitable for measuring the velocity profile by this sensor, the measured velocity profile agrees well with the theoretical velocity profile, as indicated by the broken line in Figure 7 . Because the model vessel was elliptically deformed by its own weight or by the high pressure drop in the tube caused by high viscous blood flow, the distance that the probe was translated was $0.9 \mathrm{~mm}$ shorter than the tube inner diameter. Therefore, the theoretical flow velocity profile was calculated by assuming that the tube diameter was $5.1 \mathrm{~mm}$ and that the flow was Poiseuille flow. The measured velocity profile of 

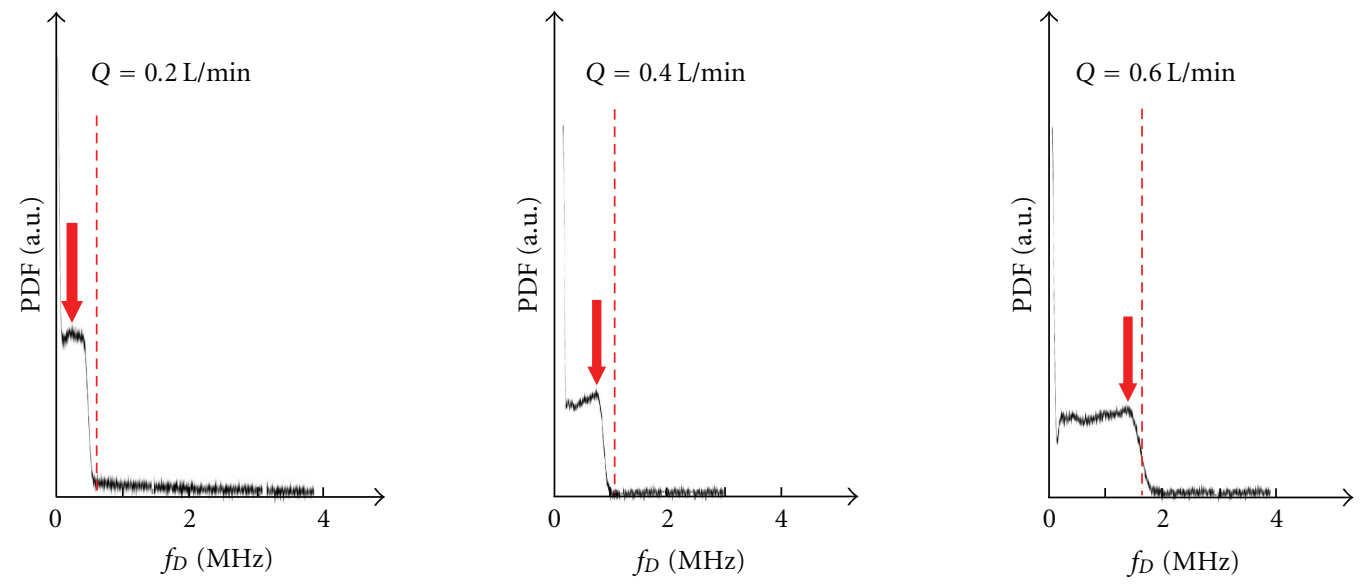

(a) Caprine blood $\left(H_{\mathrm{ct}}=40 \%\right.$, He-Ne laser $)$
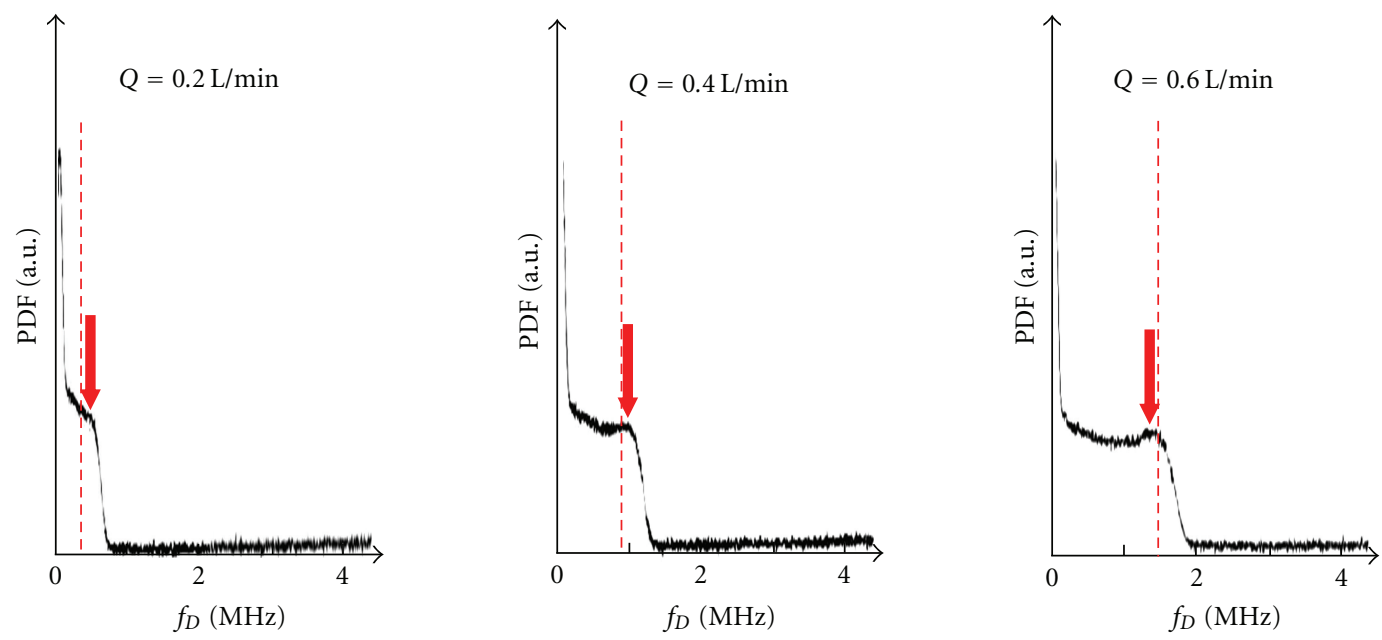

(b) Caprine blood ( $H_{\mathrm{ct}}=40 \%$, laser diode $)$
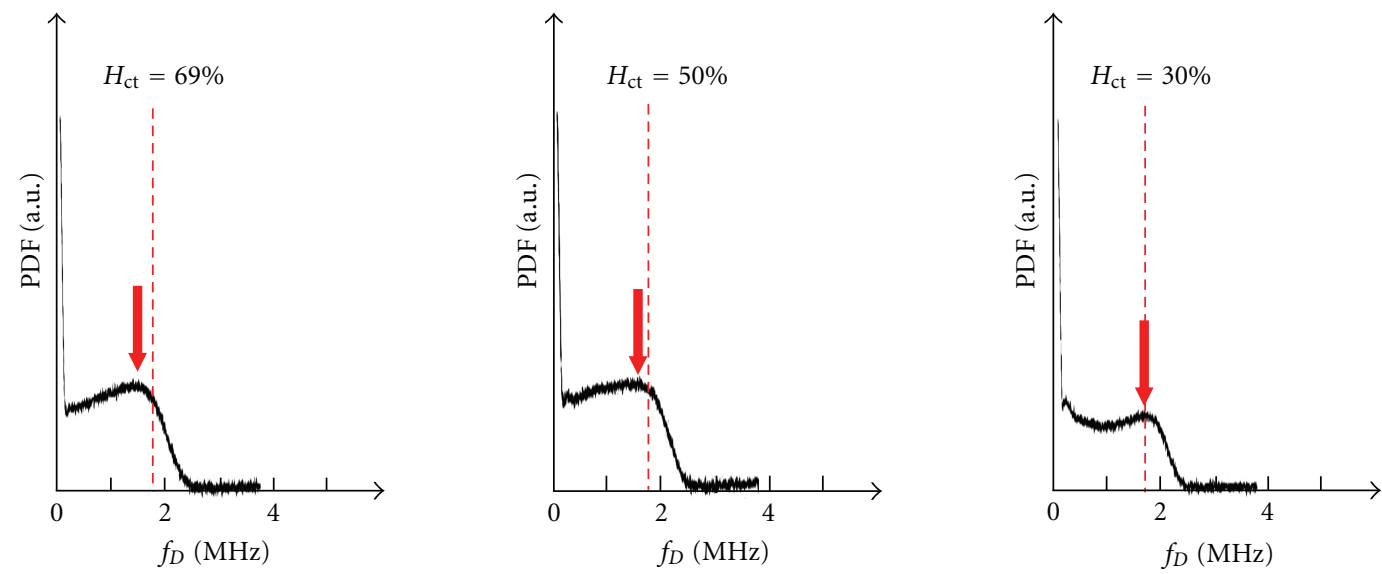

(c) Bovine blood ( $Q=0.6 \mathrm{~L} / \mathrm{min}$., He-Ne laser)

FIGURE 8: PDFs of Doppler signals using needle LDV system.

human whole blood flow was slightly rounded at the center of the tube, but the influence of a partial plug flow was not observed. These results demonstrate that this fiber-optic LDV system is able to accurately measure the local velocity of human whole blood flow.
3.2. Influence of Laser Wavelength and Blood Concentration on Measurement Accuracy. This study used a helium-neon laser as a monochromatic light source for the LDV system. However, light with a wavelength in the range 700-1200 nm can easily penetrate living tissue [7]. In a previous study 


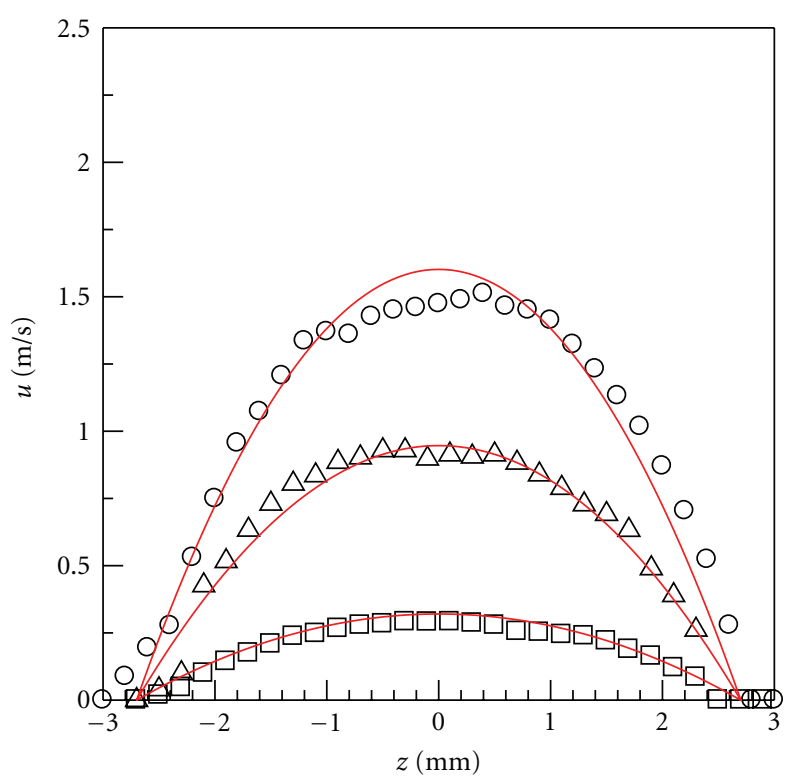

Caprine blood $H_{\mathrm{ct}}=40 \%$
- Theory
$\triangle Q=0.6 \mathrm{~L} / \mathrm{min}$
○ $Q=1 \mathrm{~L} / \mathrm{min}$
$\square Q=0.2 \mathrm{~L} / \mathrm{min}$

(a) Using He-Ne laser

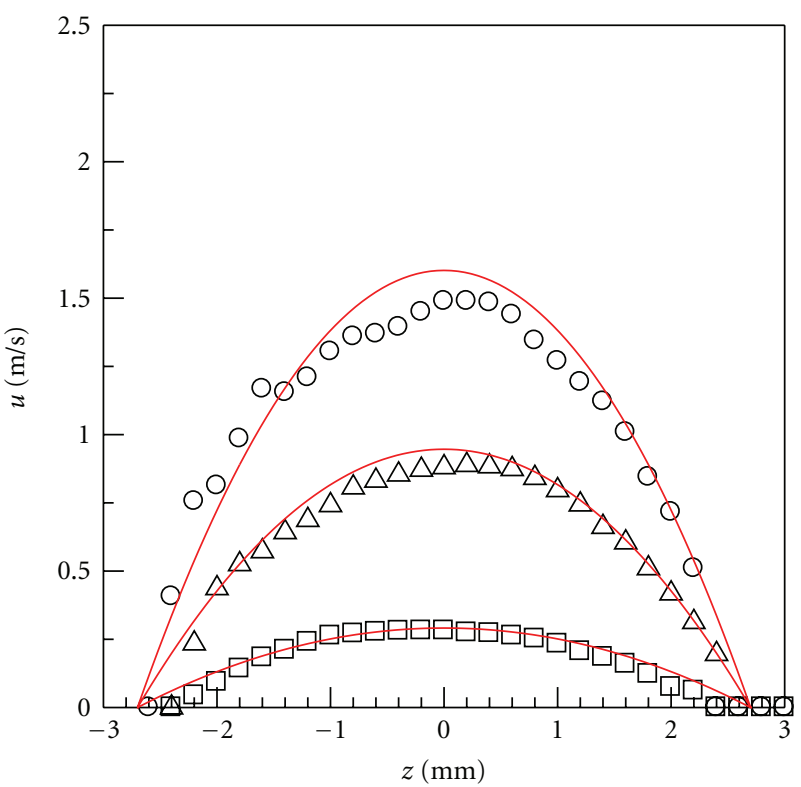

Caprine blood $H_{\mathrm{ct}}=40 \%$

$\begin{array}{ll}\text { Theory } & \triangle Q=0.6 \mathrm{~L} / \mathrm{min} \\ Q=1 \mathrm{~L} / \mathrm{min} & \square Q=0.2 \mathrm{~L} / \mathrm{min}\end{array}$

(b) Using laser diode $(\lambda=830 \mathrm{~nm})$

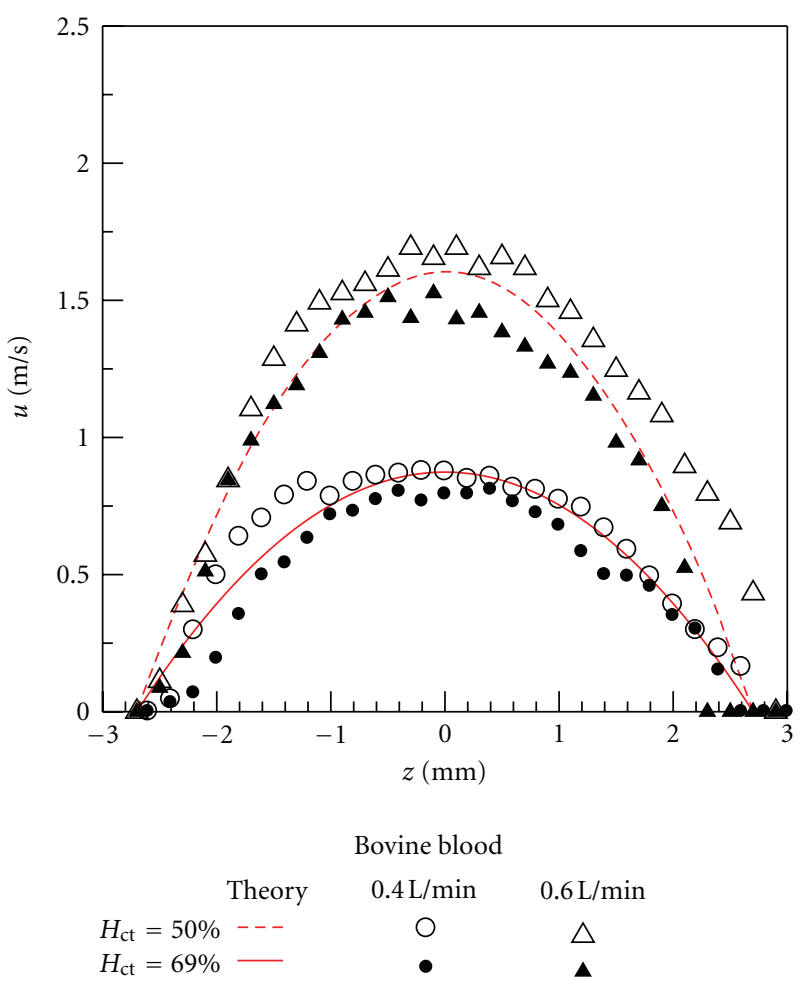

(c) Influence of hematocrit

Figure 9: Blood flow velocity profiles in model vessel. 
in which we measured the optical transmittance of whole bovine blood, we found that light with wavelengths of 830 and $632.8 \mathrm{~nm}$ from a normal-cut fiber tip decayed in intensity to 13.5 and $2.5 \%$, respectively, at a distance of $L=$ $220 \mathrm{~nm}$ from the fiber tip [8]. Because this fiber-optic probe has both transmission and receiving optical components and the light scattered from particles (e.g., red blood cells) is collected by the same fiber-optic probe, the maximum intensities of the light collected by the fiber are expected to be, respectively, $1.8 \%$ (LD: $\lambda=830 \mathrm{~nm}$ ) and $0.06 \%$ (He-Ne: $\lambda=632.8 \mathrm{~nm}$ ) of the light from the fiber tip. This will affect the results of velocity measurements. Therefore, the effects of the blood concentration and wavelength dependence of the LDV system were studied.

Whole caprine blood consisting of $40 \%$ hematocrit and concentrated bovine blood consisting of $69 \%$ hematocrit were used in this experiment. A helium-neon laser $(\lambda=$ $632.8 \mathrm{~nm}$; power: $25 \mathrm{~mW})$ and a laser diode $(\lambda=830 \mathrm{~nm}$; power: $30 \mathrm{~mW}$ ) were used as light sources for the LDV system. The wavelength dependencies of the half wave plate and the polarized beam splitter in the fiber-optic LDV system were corrected. However, the wavelength dependence of the photomultiplier tube was not corrected.

Figure 8 shows an example of a Doppler frequency spectrum obtained at the center of the test tube. The vertical axis shows the PDF of the time-averaged Doppler signal, while the horizontal axis shows the frequency. The vertical broken lines in each spectrum indicate the Doppler frequency corresponding to the real velocity calculated from the volumetric flow rate under the assumption of Poiseuille flow. The arrows in the graphs indicate the reading frequencies. Figure 9 shows the results of the velocity profile measurements.

These results show that the pedestal noise component in the frequency spectrum was slightly lower for the heliumneon laser than for the laser diode. However, the wavelength difference did not appear to affect the results. This is ascribed to differences between the blood samples and to the heliumneon laser beam being more coherent than the laser diode beam. At both wavelengths, a peak was observed in each spectrum obtained. In addition, this fiber-optic LDV system could measure the flow velocity of high concentrated blood (69\% hematocrit). The measured velocity profiles of each blood flow were slightly more rounded in the center of the tube than a parabolic velocity profile. This flow in the center of the tube is known as partial plug flow and is observed in for non-Newtonian flow [9]. The above results demonstrate that this fiber-optic LDV sensor can measure blood flow velocities of blood samples with higher concentrations than whole blood.

3.3. Influence of Insertion Angle on Measurement Accuracy. To determine the frequency shift produced the Doppler effect, the flow velocity vector must have a component parallel to the incident laser beam. Therefore, in a previous study [6], we inserted the sensor probe diagonally into the working fluid. However, the effect of the insertion angle on the measurement accuracy of the catheter LDV system has not

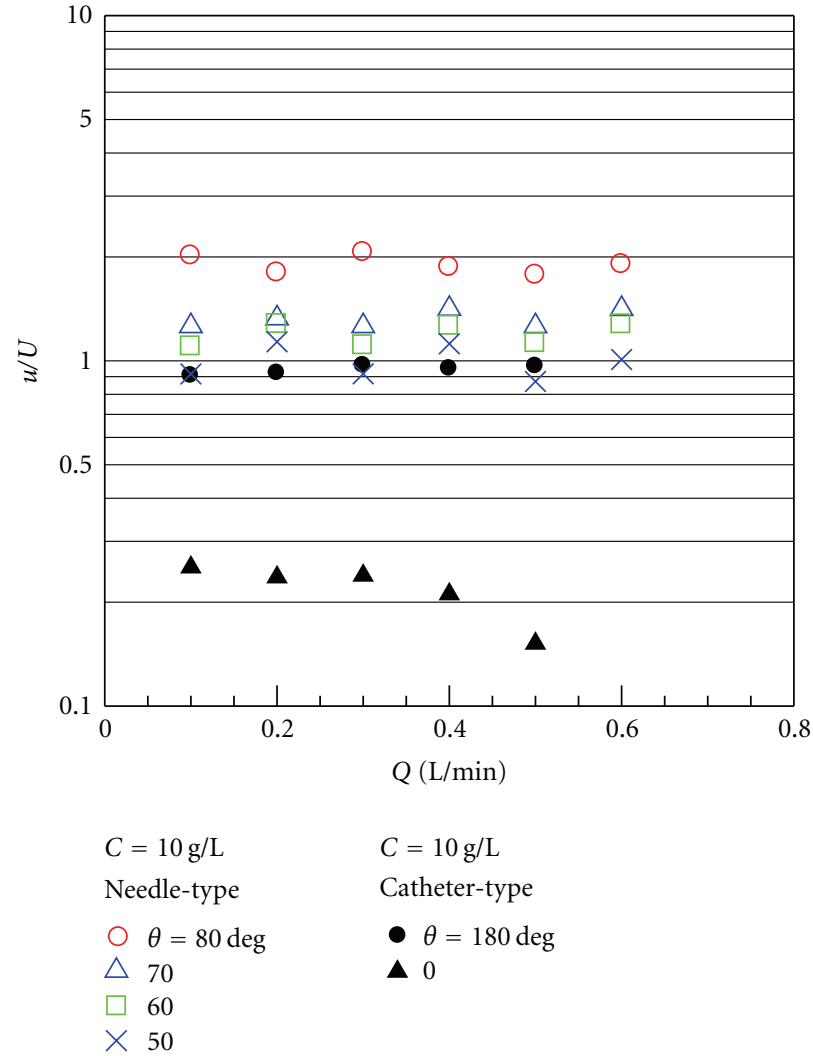

FIGURE 10: Relationship between injection angle and error ratio of measured velocity.

been determined. Therefore, the influence of the insertion angle on the measurement accuracy was investigated in the present study. The axial flow velocity was measured using catheter and needle LDV systems for various insertion angles in a white pigment suspension, which was a model blood.

Figure 10 shows the relationship between the volumetric flow rate and the error ratio (defined as the ratio of the measured velocity in the center of the tube to theoretical velocity obtained from volumetric flow rate) for various insertion angles when the white pigment concentration was $C=10 \mathrm{~g} / \mathrm{L}$. Figure 11 shows examples of spectra obtained of the Doppler signal using the catheter LDV system. The vertical broken lines and the arrows in each spectrum indicate the same parameters as above. When the white pigment concentration was lower than $C=24 \mathrm{~g} / \mathrm{L}$, a clear peak was observed in spectra obtained using the catheter LDV system when $\theta=180^{\circ}$; it was almost same as the theoretical velocity calculated from the flow rate.

In contrast, when the insertion angle was $\theta=0^{\circ}$, the frequency peaks in the spectra disappeared when $C=$ $10 \mathrm{~g} / \mathrm{L}$ and the obtained Doppler frequencies were much lower than the estimated frequencies corresponding to axial velocity in the tube. This is considered to be because the measurement point was located in the wake flow generated by the boundary layer flow in the fiber. The measurement accuracy of the needle LDV system was reduced when the insertion angle was close to $90^{\circ}$. This result indicates that the 


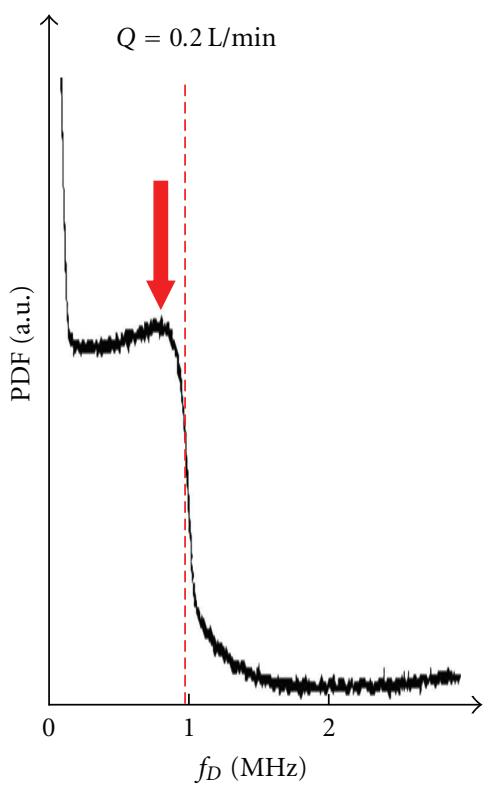

(a) $\theta=180 \mathrm{deg}$. $(C=24 \mathrm{~g} / \mathrm{L})$

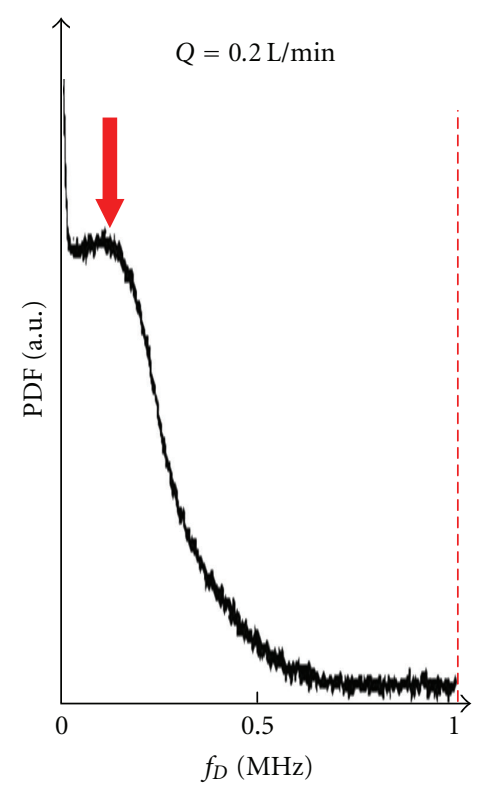

(b) $\theta=0$ deg. $(C=10 \mathrm{~g} / \mathrm{L})$

FIGURE 11: PDFs of Doppler signals obtained using catheter LDV.

setting error of the insertion angle affects the frequency-tovelocity conversion using (1). The optimal insertion angles are considered to be $\theta=50^{\circ}$ and $180^{\circ}$ for the needle and catheter LDV systems, respectively. The catheter LDV system is the preferred insertion method when using this sensor in clinical applications. We next performed blood flow measurements using the catheter LDV sensor.

\subsection{Measurement of Blood Flow Velocity by Catheter LDV} System. The sensor tip of the catheter LDV system was aligned upstream, and working fluids of bovine whole blood and caprine blood $\left(H_{\mathrm{ct}}=40 \%\right)$ were used. In this experiment, the bovine blood concentration was varied between 30 and $69 \%$ by adding PBS. The working fluid was pumped through the circuit (see Figure 3). A helium-neon laser and a laser diode were used as the oscillators in this experiment.

Figure 12 shows examples of frequency spectra obtained in blood flow measurements using the catheter LDV system. No clear peak of the Doppler frequency corresponding to the flow velocity was observed in any of the spectra. In addition, the signal-to-noise ratio was significantly lower than for the needle LDV system; the peak becomes broader with increasing blood concentration. This result indicates that multiple scattering occurred between the fiber tip and the focal point of the fiber. Thus, we modified the definition of the measurement frequency: the Doppler frequency was taken to be the frequency at which the spectral profile increased steeply. The measurement frequencies are indicated by arrows with dotted lines in Figure 12. Figures 13 and 14 show the results of converting this frequency to velocity using (1). Figure 13 shows the effect of using different light sources and the error ratio of the measurement velocity for the catheter LDV system, while Figure 14 shows the relationship between hematocrit of bovine blood and the error ratio for velocities measured using a catheter LDV system.

Most velocities measured in this experiment were within several percent of the theoretical values along the tube axis. However, the measured velocities for caprine blood flow (40\% hematocrit) using the laser diode were about 3-15\% lower than the theoretical values along the tube axis. Since the pedestal noise component in each spectrum is higher for the laser diode than for the helium-neon laser, this result suggests that the coherence of the laser light affects the signal intensity. It indicates that light coherence is an important factor when measuring blood flow velocity using light transmission. Consequently, the measurement accuracy was higher for the helium-neon laser than for the laser diode.

\section{Conclusions}

A fiber-optic laser Doppler velocimetr system was developed using a chemically etched optical fiber with a convex lens-like tip as the light transmitting and receiving probe. The fiberoptic LDV system was used to measure the local flow velocity in opaque and semiopaque fluids, including a white pigment suspension, whole human blood, whole bovine blood, and whole caprine blood. The performance of this system was evaluated and the following results were obtained.

(1) The velocity distribution across the vessel could be very accurately measured for flows of whole human blood, whole caprine blood, and concentrated bovine blood (hematocrit 69\%).

(2) The light source (a He-Ne laser and a laser diode) had little effect on the measurement accuracy. 

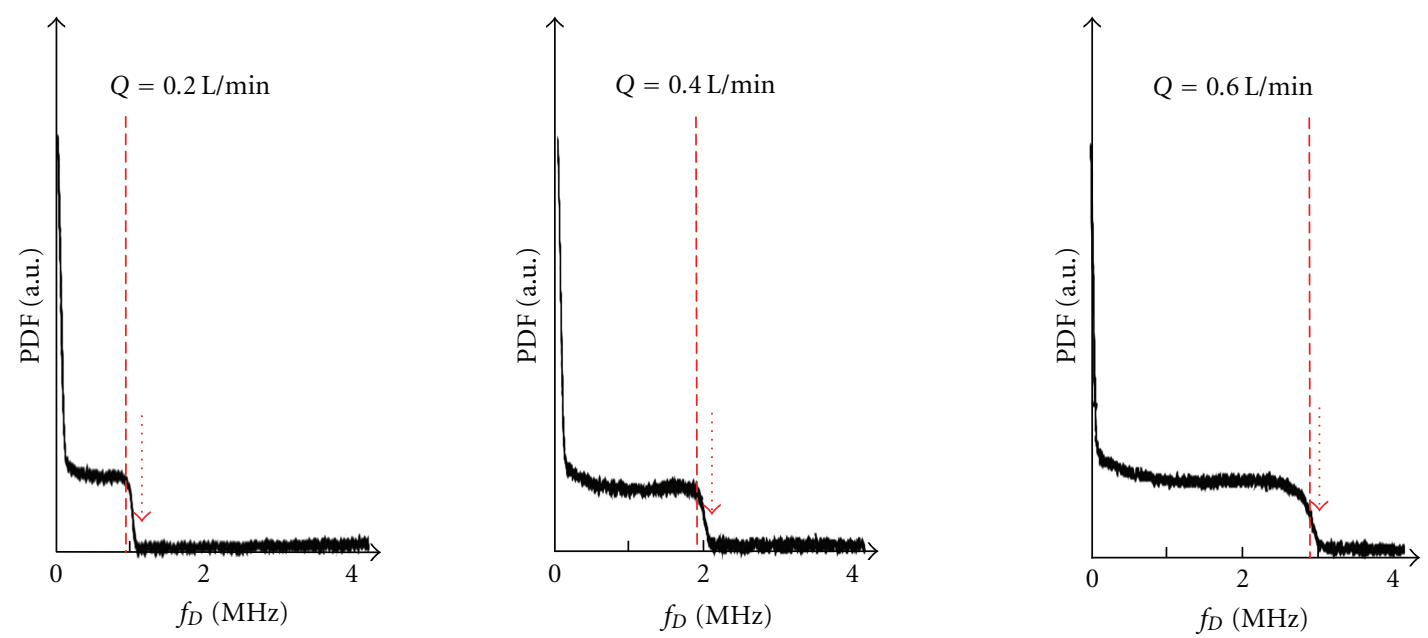

(a) Caprine blood $\left(H_{\mathrm{ct}}=40 \%\right)$, He-Ne laser
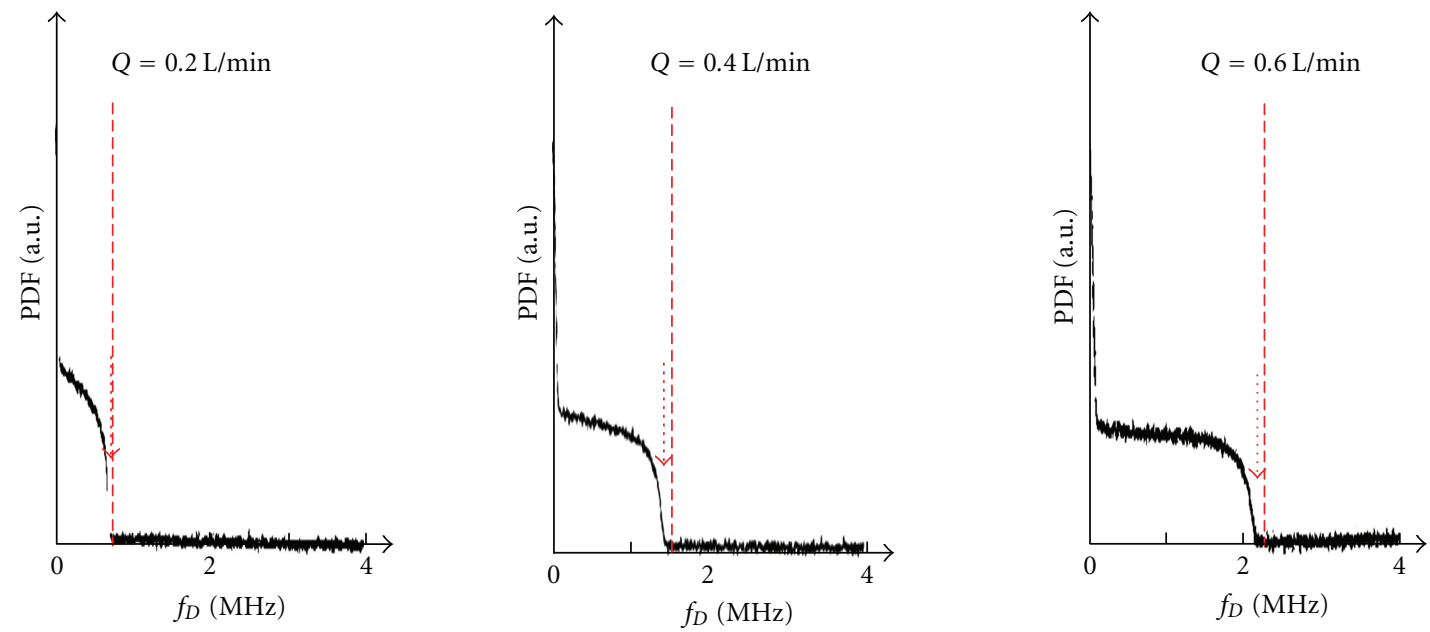

(b) Caprine blood $\left(H_{\mathrm{ct}}=40 \%\right)$, laser diode
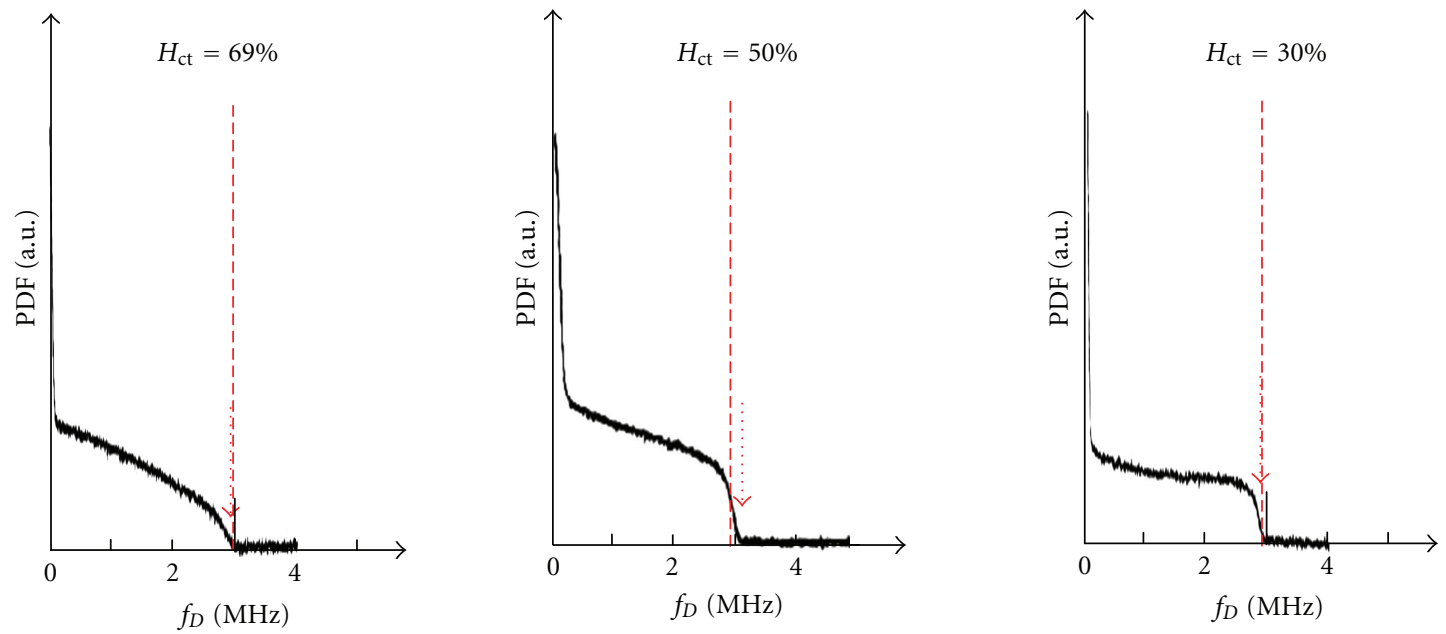

(c) Bovine blood, $Q=0.6 \mathrm{~L} / \mathrm{min}$., He-Ne laser

FIGURE 12: PDFs of Doppler signal using catheter LDV system. 


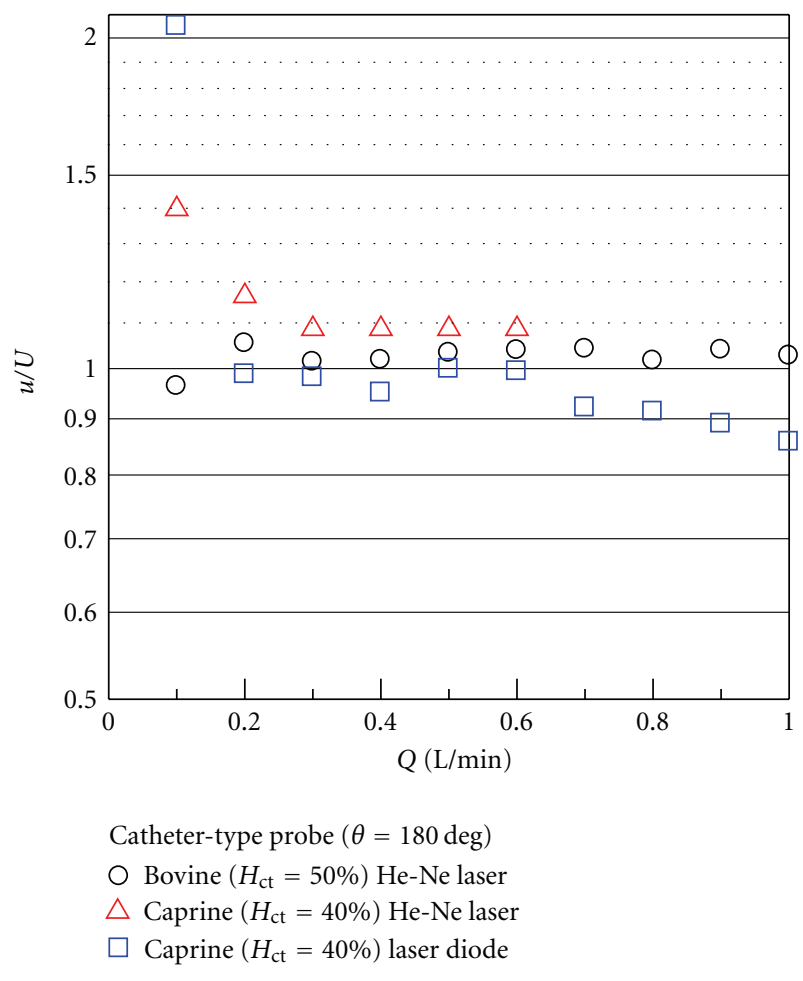

Figure 13: Effect of different light sources and error ratio of measured velocity for catheter LDV system.

(3) The insertion angle of the fiber into the flow significantly affects the measurement accuracy; an insertion angle of about $50^{\circ}$ is optimal for the injection needle LDV system.

(4) For the catheter LDV system, an insertion direction against the flow is better than one with the flow due to the effect of the wake behind the fiber tip.

\section{Nomenclature}

T: $\quad$ Etching time (h)

$r: \quad$ Radial position relative to fiber axis $(\mu \mathrm{m})$

$L: \quad$ Distance from fiber tip $(\mu \mathrm{m})$

$z$ : Distance from center of the tube to upper vertical $(\mathrm{mm})$

Q: Volumetric flow rate $(\mathrm{L} / \mathrm{min})$

$u$ : Local flow velocity $(\mathrm{m} / \mathrm{s})$

$U$ : Axial flow velocity calculated from volumetric flow rate $(\mathrm{m} / \mathrm{s})$

$C$ : Mass concentration of white pigment suspension $(\mathrm{g} / \mathrm{L})$

$H_{\mathrm{ct}}$ : Hematocrit (volumetric concentration of erythrocyte in blood) (\%)

$f_{D}$ : Doppler shift frequency $(\mathrm{MHz})$

$\theta: \quad$ Fiber insertion angle relative to flow direction $\left(^{\circ}\right)$

$\lambda$ : $\quad$ Laser wavelength $(\mathrm{nm})$.

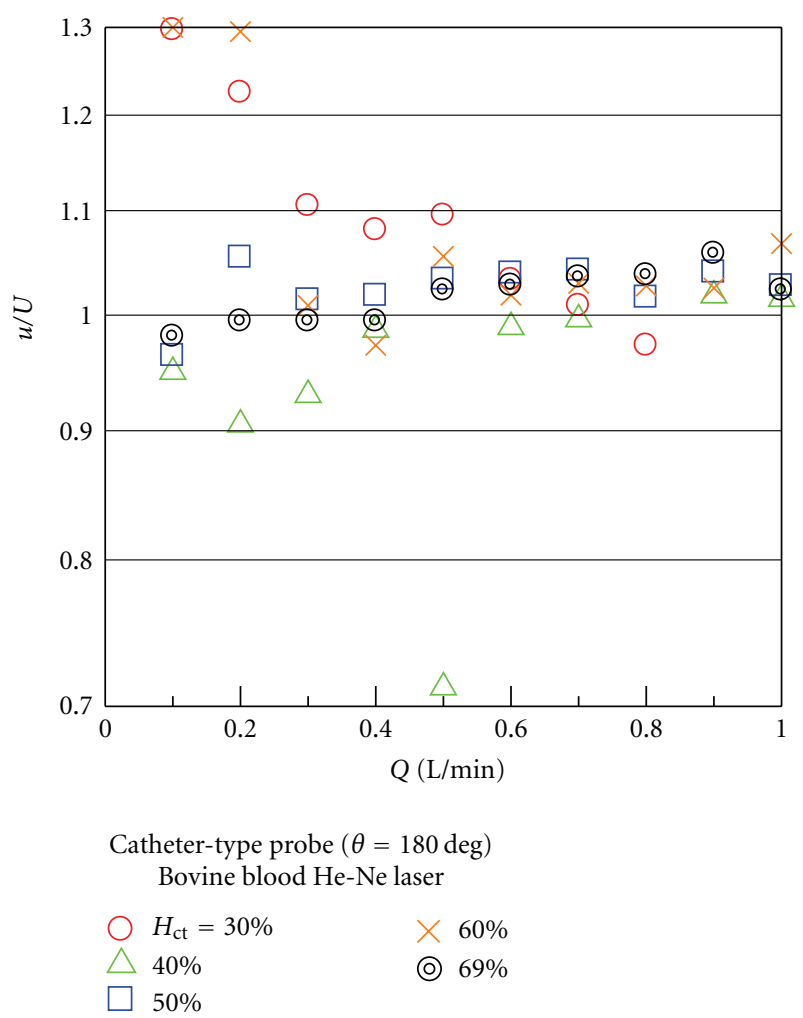

FIGURE 14: Relationship between hematocrit of bovine blood and error ratio of measured velocities using catheter LDV system.

\section{Acknowledgments}

A part of this study was supported by the Ministry of Education, Culture, Sport, Science and Technology (MEXT), Japan, through the "Academic Frontier" Project at Kansai University on "Creation of Realistic Models of Human Tissues, Organs using Nano/Sub-micro Technology and their Development to Artificial Tissues/Organs", 2003-2007. The authors express their appreciation to the Japanese Red Cross Osaka Blood Center and the Department of Artificial Organs of the National Cardiovascular Center for providing blood samples.

\section{References}

[1] T. Tanaka and G. B. Benedek, "Measurement of the velocity of blood flow (in vivo) using a fiber optic catheter and optical mixing spectroscopy," Applied Optics, vol. 14, no. 1, pp. 189196, 1975 .

[2] F. Kajiya, N. Hoki, G. Tomonaga, and H. Nishihara, "A laserDoppler-velocimeter using an optical fiber and its application to local velocity measurement in the coronary artery," Experientia, vol. 37, no. 11, pp. 1171-1173, 1981.

[3] K. Ohba and T. Matsuno, "Local velocity measurement of opaque fluid flow using laser Doppler velocimeter with optical dual fiber pickup," Transactions of the Japan Society of Mechanical Engineers, Series B, vol. 49, no. 447, pp. 2380-2389, 1983.

[4] K. Ohba and N. Fujiwara, "Development of fiber optic laser Doppler velocimeter for measurement of local blood velocity," 
in Proceedings of the 5th International Conference on Laser Anemometry, vol. 2052 of Proceedings of SPIE, pp. 195-201, Koningshof, The Netherlands, August 1993.

[5] K. Ohba and M. Nishiyama, "Development of small fiber optic laser Doppler velocimeter sensor for measurement of local blood velocity," Japanese Journal of Medical Electronics and Biological Engineering, vol. 36, supplement 1, p. 307, 1998 (Japanese).

[6] T. Tajikawa, M. Takeshige, W. Ishihara, S. Kohri, and K. Ohba, "Development of miniaturized fiber-optic laser Doppler velocimetry sensor for measurement of local blood velocity (fabrication of convex or concave lens-like fiber tip and the characteristics of sensor optical system)," Journal of Fluid Science and Technology, vol. 4, no. 1, pp. 62-74, 2009.

[7] K. Shimizu and K. Yamamoto, "Biomedical imaging by optical computer tomography-Status of basic research and CT Imaging with CW laser," BME, vol. 8, no. 8, pp. 4-13, 1994 (Japanese).

[8] K. Ohba and T. Matsuno, "Development of fiber optic laser Doppler velocimeter sensor for measurement of local blood velocity," Journal of Flow Measurements, vol. 8, pp. 43-46, 1995 (Japanese).

[9] Y. C. Fung, Biomechanics: Mechanical Properties of Living Tissues, Springer, 1981. 

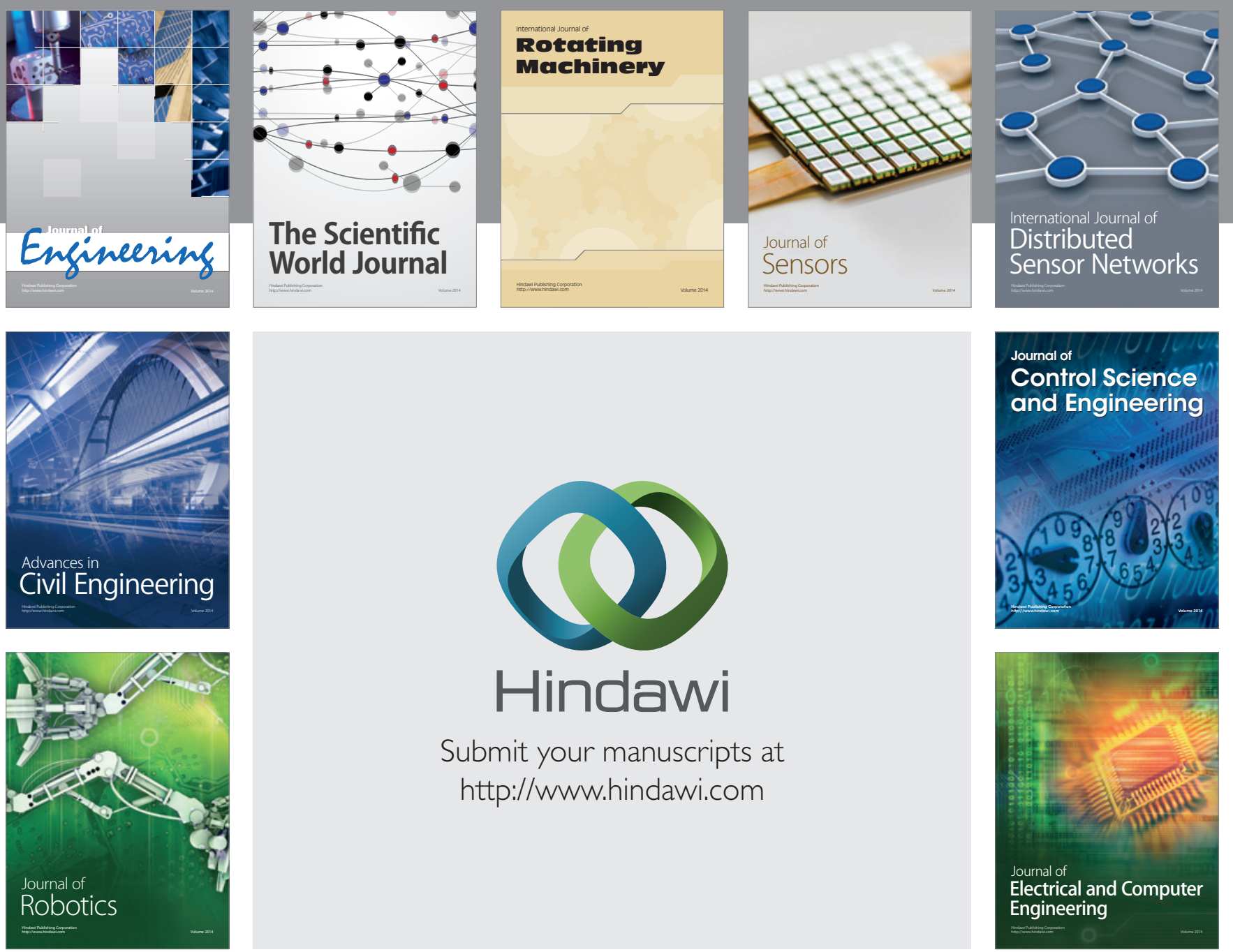

Submit your manuscripts at

http://www.hindawi.com
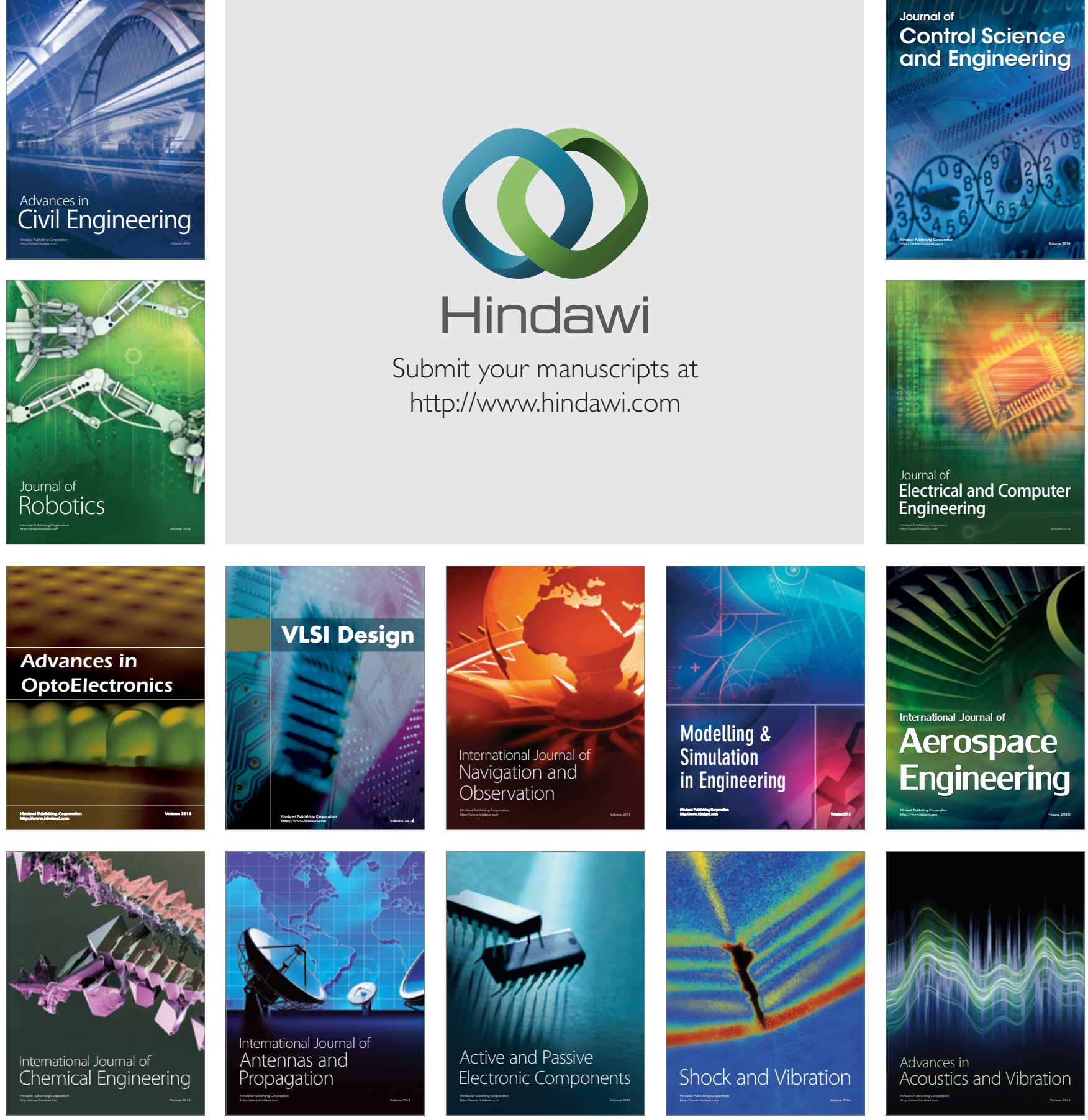